\title{
Improving response time in traf fic police infraction registration system
}

\begin{abstract}
Advanced of civilian wireless network infrastructure made the mobile devices increasingly popular. One of the main police requirements is information systems to provide information for police anywhere and anytime. Mobile equipments are widely used for communication but lacking of security makes it unsuitable for transmitting the confidential data. In this paper, we discuss requirements of the traffic police data center firstly, and then propose a solution for improving response time of traffic police data center. Policemen in the system can use various kinds of mobile terminals, such as mobile phones, tablet PCs, personal digital assistants, and laptops equipped with wireless LAN (WLAN) or General Packet Radio Service (GPRS) device to access data in the central database. The system addresses the requirement of security and transaction speed, and can be extended to the other mobile information systems such as e-commerce.
\end{abstract}

